\title{
Retenção de conhecimento em serviços
}

Rodrigo Valio Dominguez Gonzalez

Doutor e mestre em Engenharia de Produção pela Universidade Federal de São Carlos. Professor da Universidade de Campinas (UNICAMP)

http://dx.doi.org/10.1590/1981-5344/2352

Atualmente, o desafio para os pesquisadores e gestores na área da gestão do conhecimento é estudar modelos que promovam a aquisição, retenção, distribuição e utilização do conhecimento por indivíduos e grupos das organizações. As empresas prestadoras de serviço são exemplos de organizações que apresentam dificuldades na retenção do conhecimento, uma vez que este é criado nos locais de prestação de serviço, isto é, distante da sede da empresa. Quando a empresa prestadora de serviço não se estrutura internamente a fim de resgatar este conhecimento diluído entre vários locais de operação, a chance de ocorrer a perda do conhecimento e de vantagem competitiva é grande. Desta forma, o principal objetivo deste trabalho é analisar um modelo organizacional voltado para um prestador de serviço que promova a retenção do conhecimento adquirido em seus diversos clientes. Os resultados apontam que as empresas prestadoras de serviço devem focar seu processo de retenção de conhecimento em um departamento específico. Este departamento tem a tarefa de identificar e resgatar as melhores práticas e lições aprendidas entre todos os funcionários que atuam em diferentes clientes em bancos de dados, e, além disso, promover a integração desses funcionários a fim de promover a distribuição de conhecimento tácito.

Palavras-chave: Retenção do conhecimento; Distribuição do conhecimento; Recuperação do conhecimento; Gestão do conhecimento; Prestação de serviços.

\section{Knowledge retention in services}

Currently, the challenge for researchers and managers in the area of knowledge management is to study models 
that promote the acquisition, retention, distribution and use of knowledge by individuals and groups working in organizations. The service companies are examples of organizations that have difficulties in knowledge retention process, once it is created and acquired in different service sites, i.e., distant from the company headquarters. When the service provider company did not mobilize and structure internally in order to retain this knowledge diluted across multiple sites, the chance of loss the knowledge and competitive advantage is great. Thus, the main objective of this paper is to analyze an organizational model focused to a service provider that promotes the retention of the knowledge acquired in their different clients. The results suggest that service companies should focus on the process of knowledge retention in a specific department. This department has the task to identify and storage best practices and lessons learned among all employees who work on different clients in databases, and in addition, to promote the integration of these employees in order to promote the distribution of tacit knowledge.

Keywords: Knowledge retention; Knowledge distribution; Knowledge retrieval; Knowledge management; Service.

Recebido em 05.03.2015 Aceito em 10.12.2015

\section{Introdução}

A Gestão do Conhecimento (GC) não é um assunto novo e, atualmente, existem vários modelos para esta finalidade. Em contraste, as disciplinas específicas que cercam o tema GC ainda requerem muito desenvolvimento por parte dos pesquisadores (DURST; EDVARDSSON, 2012).

A retenção do conhecimento pode ser considerada uma subdisciplina chave relacionada ao processo de GC. A importância da retenção do conhecimento se dá pelo fato de que a perda de conhecimento pode reduzir a vantagem competitiva e colocar uma organização em dificuldades econômicas (MARTINS; MEYER, 2012; ARIF et al., 2009.).

É consenso na pesquisa sobre a retenção do conhecimento de que a saída de funcionários de uma organização pode levar à perda do conhecimento organizacional. DeLong e Davenport (2003) apontam que um alto nível turnover dentro de uma organização pode gerar uma "amnésia operacional e institucional perigosa". Além disso, a condição de envelhecimento da população e aposentadoria das pessoas ativas pode levar as organizações perderem uma parte do conhecimento referente ao trabalho específico (NICHOLSON, 2008). Dado que as organizações não 
podem perder o conhecimento especializado, elas precisam encontrar maneiras de reter o conhecimento na mente das pessoas e dentro da organização. Se o conhecimento não é retido, as organizações não são capazes de aprender com as experiências passadas, tendo que se reinventar continuamente (MCADAM; MOFFETT; PENG, 2012; DU PLESSIS, 2003).

As empresas de serviços, por sua vez, são caracterizadas como empresas intensivas em conhecimento. Assim, o conhecimento e as habilidades dos funcionários se destacam ainda mais em relação a qualquer outro ativo organizacional (MCADAM; MOFFETT; PENG, 2012; EVANSCHTIZKY et al., 2007). Nem todo o conhecimento necessário para prestar um serviço pode ser desenvolvido e acumulado em uma única empresa (EDVARDSSON; OSKARSSON, 2011). Em cada site de prestação de serviços é desenvolvida uma vasta gama de conhecimentos e competências. Quando a organização não consegue gerenciar esse conhecimento, diluído entre vários sites, ela torna-se menos competitiva e perde uma parte significativa de seus ativos.

A revisão da literatura revela que poucas pesquisas têm sido realizadas na área de retenção do conhecimento organizacional com foco no indivíduo, equipe e modelo organizacional para a retenção do conhecimento. Vários estudos sobre a retenção do conhecimento focam este tema sob a perspectiva da tecnologia da informação (TI), com foco no conhecimento explícito da organização. No entanto, estudos sobre GC devem ser sensível ao fato de que o conhecimento tácito é mais difícil de imitar, é capaz de gerar uma vantagem competitiva e não pode ser armazenado em bases de dados (SPENDER, 1996; GRANT, 1996; NICKERSON; ZENGER, 2004). Assim, este artigo tem como objetivo principal analisar o modelo organizacional aplicado em uma empresa prestadora de serviços que é capaz de reter o conhecimento adquirido em diferentes clientes e distribuí-lo entre os vários funcionários e grupos de empresa.

Além desta introdução, o artigo está estruturado em outras quatro seções. A próxima seção aborda o referencial teórico utilizado para fundamentar a pesquisa de campo. Em seguida, é apresenta a metodologia adotada para a pesquisa de campo. Posteriormente, é realizada a descrição e análise do caso de uma empresa prestadora de serviços. E, finalmente, são realizadas as considerações finais sobre o problema de pesquisa.

\section{Revisão da literatura}

\subsection{Retenção do conhecimento}

Segundo Polanyi (1967), existe dois tipos de conhecimento, um tácito e outro explícito. E extremamente importante o entendimento sobre o significado de conhecimento tácito e explícito a fim de adotar iniciativas para a retenção do conhecimento. Se a parte explícita do conhecimento é mais valorizada, a empresa pode minimizar o processo de retenção a 
Tecnologia da Informação (TI). No entanto, quando as duas parcelas de conhecimento são abordados de uma forma coordenada e conjunta, a organização pode obter vantagem competitiva sustentável (TSOUKAS, 1996).

O conhecimento explícito é considerado, de acordo com Smedlund (2008), como a melhor maneira de transferir conhecimento. No entanto, esta característica torna-se suscetível a imitação por parte dos concorrentes, tornando-o frágil como a única fonte de vantagem competitiva organizacional devido ao seu baixo grau de apropriabilidade (NELSON; WINTER, 1982). O conhecimento tácito, por sua vez, é prático, intrínseco às pessoas, transferido através de relações sociais, sendo específico ao contexto, portanto, pode ser considerado de difícil formulação e comunicação. É através deste conhecimento que a organização pode gerar inovação e novos conhecimentos (SMEDLUND, 2009; NELSON; WINTEr, 1982). Tendo em vista que o conhecimento tácito está intimamente relacionado com a capacidade humana em realizar tarefas, este conhecimento tem sido utilizado para apoiar as definições de competências essenciais e capacidades organizacionais (MOLINA; AMADO; AROSTEGUI, 2010).

A fim de desenvolver uma análise do processo de retenção do conhecimento, é necessário definir o seu escopo. Retenção do conhecimento consiste em três atividades: aquisição, armazenamento e recuperação do conhecimento (WALSH; UNGSON, 1991).

A aquisição refere-se ao processo intraorganizacional que facilita a criação de conhecimento tácito e explícito, partindo dos indivíduos e integrando-se ao nível organizacional, bem como a identificação e absorção de informação e conhecimento de origem externa (FILIPPINI et al., 2012; HUBER, 1991), portanto, esta pesquisa parte da premissa que a aquisição consiste na criação de conhecimento dentro da organização por meio de um processo de aprendizagem, e também na aquisição de conhecimento externo, originado da ação associativa com outras organizações, consultorias e universidades. O processo criativo dentro de uma organização começa a partir do momento em que o conhecimento é identificado como uma solução para um problema (GONZALEZ; MARTINS, 2014).

A etapa de armazenamento do conhecimento se refere ao processo pelo qual o conhecimento é formalmente armazenado em sistemas de memória física e informalmente mantido na forma de valores, normas e crenças (CLAVER; ZARAGOZA; QUER, 2007; ALAVI; LEIDNER, 2001). Lin (2007) explica que o armazenamento do conhecimento implica em um processo de conversão, envolvendo a organização, a estruturação, o armazenamento e, por fim, a combinação do conhecimento a fim de facilitar o uso futuro por parte dos interessados. Assim, TI aparece como ferramenta chave nesta fase, oferecendo três aplicações: (i) a codificação e o compartilhamento do conhecimento; (ii) a criação de diretórios corporativos do conhecimento; (iii) a criação de redes do conhecimento (ALAVI; LEIDNER, 2001). 
Todavia, autores como Rowley (2001), Thompson e Walshman (2004) e Huysman e Wulf (2006) atenuam a importância da TI na fase de retenção do conhecimento. Huysman e Wulf (2006) explicam que as organizações precisam criar uma cultura que encoraje o compartilhamento do conhecimento. Segundo Rowley (2001), nem todo o conhecimento tácito precisa, necessariamente, ser transformado em explícito, uma vez que estes dois tipos de conhecimento são complementares e interdependentes (INAZAWA, 2009; GAO; LI; CLARKE, 2008). Rowley (2001, p. 234) ainda pondera: "Not all tacit knowledge needs to be made explicit; the organization needs to develop a shared understanding of which types of knowledge need to be articulated and shared".

A recuperação do conhecimento é o processo pelo qual novas informações a partir de diferentes fontes são compartilhadas e que, eventualmente, podem conduzir à criação de novos conhecimentos e informações (GAMMELGAARD; RITTER, 2005). No entanto, de acordo com Lee e Yang (2000, p. 790), este processo de compartilhamento requer 0 desenvolvimento de um 'ambiente de compartilhamento': "The most effective way to disseminate knowledge and best practices is through systematic transfer. This is, to create a knowledge sharing environment $[\ldots]^{\prime \prime}$.

O conhecimento explícito pode ser compartilhado através de sistemas de TI, mas também por meio da interação social das pessoas (ARGOTE; MCEVELY; REAGANS, 2003). As pessoas devem contribuir a fim de que o conhecimento se integre a uma 'rede de conhecimento' e a TI, por si só, não destitui barreiras importantes para a GC. Conforme Lee e Yang (2000), a TI não altera o comportamento das pessoas, não aumenta - comprometimento gerencial, e nem cria um entendimento compartilhado entre as pessoas.

Para que seja utilizado, o conhecimento necessariamente já sofreu a ação das duas fases anteriores do processo de GC, ou seja, o conhecimento já foi adquirido (construído) e armazenado, de forma tácita nos indivíduos ou explícita em documentos, bancos de dado, entre outros. Freeze e (2007) identificam quatro formas em que o conhecimento pode ser encontrado na organização (Tabela 1) e cada um destes estados apresentam formas específicas de distribuição.

Tabela 1 - Modos de distribuição do conhecimento

\begin{tabular}{lll}
\hline Tipo & Natureza & Origem \\
\hline Expertise & Tácita & Experiência humana \\
Lições aprendidas & Tácita / Implícita & Front-line \\
Documentos & Explícita & Relatórios (bottom-up) \\
Políticas e procedimentos & Implícita / Explícita & Relatórios (top-down) \\
\hline
\end{tabular}

Fonte: FREEZE; KULKARNI (2007).

O termo inglês expertise é melhor compreendido na língua portuguesa como a habilidade em desenvolver determinada tarefa. Por meio desta interpretação, verifica-se que a expertise é bastante particular ao ser humano, altamente tácito, referente a um domínio específico 
originado por meio da experiência. Sua estratégia de transferência é dada pela interação e ação colaborativa entre indivíduos e a retenção faz parte de uma estratégia de gerenciamento de competência.

Interações ou conversações entre as pessoas representam um modelo bastante simples para compartilhamento de expertise, contudo, tais iniciativas podem esbarrar em barreiras culturais. Para que ocorra a distribuição do conhecimento, a organização deve considerar uma abordagem de sistemas de distribuição sociotécnica, isto é, aliar a TI com aspectos culturais e estruturais da organização (INAZAWA, 2009).

As lições aprendidas compreendem o conhecimento ganho quando tarefas ou projetos são desenvolvidos por indivíduos, sendo também tratadas como 'melhores práticas' ou 'benchmark interno' (ALAVI; LEIDNER, 2001). Uma vez que a lição é aprendida, a organização necessita pesquisar uma estratégia a fim de tornar este conhecimento explícito, definido por Freeze e Kulkarni (2007) como documentos do conhecimento. Tais documentos podem ser estruturados na forma de relatórios técnicos, relatórios de pesquisa e publicações ou de uma maneira não estruturada na forma de diagramas, apresentações, figuras, manuais, tutoriais, etc. Assim, os documentos do conhecimento representam a forma explícita das lições aprendidas, que promovem o armazenamento e posterior utilização do conhecimento.

Grande parte do conhecimento desenvolvido é incorporada pela organização na forma de políticas e procedimentos, que representam o conhecimento institucional requerido para a operação eficiente e consistente de uma organização, tratando-se da rotina organizacional, isto é, um padrão comportamental desenvolvido pela organização que dirige a ação dos seus indivíduos (SILVA; FERREIRA, 2009; NELSON; WINTER, 1982). Contudo, Brown e Duguid (2001) argumentam que existem diferenças entre aquilo que está escrito e aquilo que realmente os indivíduos realizam. Tal aspecto se deve ao fato de que estas práticas e procedimentos não se referem simplesmente à distribuição de um conhecimento explícito. Freeze e Kulkarni (2007) e Gao, Li e Clarke (2003) explicam que existe um continuum no qual parte do conhecimento tácito é convertida para explícito. Entre estes dois extremos existe um domínio do conhecimento denominado pelos autores como implícito ou o conhecimento tácito que é potencialmente passível de explicitação, mas que ainda está incorporado apenas na expertise individual.

Estas três atividades são utilizadas para caracterizar a memória organizacional (WALSH; UNGSON, 1991; EVELETH, 2011; GRANT, 1996).

Grant (1996); Silva e Ferreira (2009) e Garicano e Wu (2012) apontam que o principal papel da firma está na integração do conhecimento especializado individual e que a coordenação hierárquica da firma falha no processo de integração, uma vez que grande parte do conhecimento é mantida no estado tácito. Grant (1996) sugere quatro tipos de mecanismos que estimulam a integração de conhecimento implícito (informal) e explícito (formal):

1 Regras que regulamentam as interações entre os indivíduos; 
2 Sequenciamento, relacionado à integração do conhecimento especializado;

3 Rotinas que sustentam padrões complexos de interação entre os indivíduos;

4 Grupos de solução de problema que promovem a interação 4 não padronizada.

Neste contexto, a firma passa a ter conotação de repositório de conhecimento, caracterizando-se como um local físico que sustenta a criação e desenvolvimento, provendo um contexto social, econômico e político (SILVA; FERREIRA, 2009; GRANT, 1996).

\subsection{Modelos da literatura que sustentam a retenção do conhecimento}

Nonaka e Takeuchi (1995) utilizam a distinção entre tácito e explícito a fim de conceberem um modelo de criação do conhecimento denominado de modelo SECI ou "espiral do conhecimento" (Figura 1). A espiral de criação de conhecimento considera que as interações da organização com o ambiente criam e proliferam o conhecimento por meio do processo de conversão de tácito para explícito.

Figura 1 - Modelo SECI de criação de conhecimento

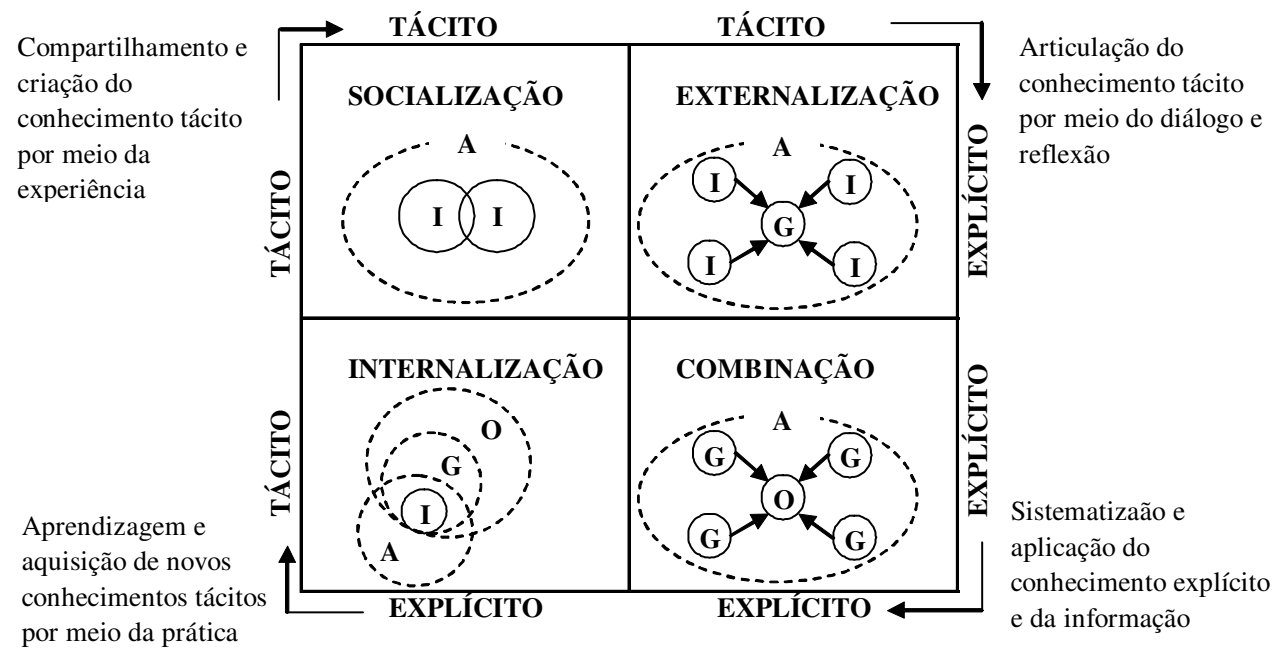

Legenda: I - Indivíduo; G - Grupo; O - Organização; A - Ambiente

Fonte: NONAKA; TAKEUCHI (1995).

O modelo propõe que o conhecimento tácito pode ser transferido entre os indivíduos por meio de um processo de socialização, e que o conhecimento tácito pode se tornar explícito através de um processo de externalização. $O$ modelo também assume que o conhecimento explícito pode ser transformado em tácito através de um processo de 
internalização, e que o conhecimento explícito pode ser transformado em outros conhecimentos explícitos através de um processo de combinação.

Nonaka e Takeuchi (1995) salientam que a criação de conhecimento por meio do modelo SECI está diretamente ligada ao desenvolvimento de espaços que promovam à interação entre os indivíduos, sendo estes denominados de 'ba' que incluem aspectos físicos e virtuais da organização que estimulam a criação de conhecimento (Figura 2).

Quatro tipos de ba são identificados dentro do processo SECI:

1 ba de origem: lugar para compartilhamento de experiências por interação face a face.

2 ba de diálogo: lugar de articulação dos modelos mentais e experiências por meio de conceitos comuns.

3 ba de sistematização: lugar de interações coletivas e virtuais.

4 ba de exercício: lugar de incorporação de conhecimento explícito por meio de interações virtuais.

A fim de garantir o acesso ao conhecimento armazenado, a memória organizacional deve ser estruturada de uma forma que sustente a recuperação do conhecimento. Walsh e Ungson (1991) argumentam que uma das razões para a dificuldade em definir a memória organizacional é que não está claro se os conceitos, que são essencialmente derivados de trabalho sobre processamento de informações em organismos biológicos, podem ser estendidos para os fenômenos sociais e organizacionais. Os autores sugerem um modelo para estruturar a memória organizacional (Figura 3), no qual o processo de retenção consiste em cinco seções principais.

Figura 2 - As quatros características do Ba

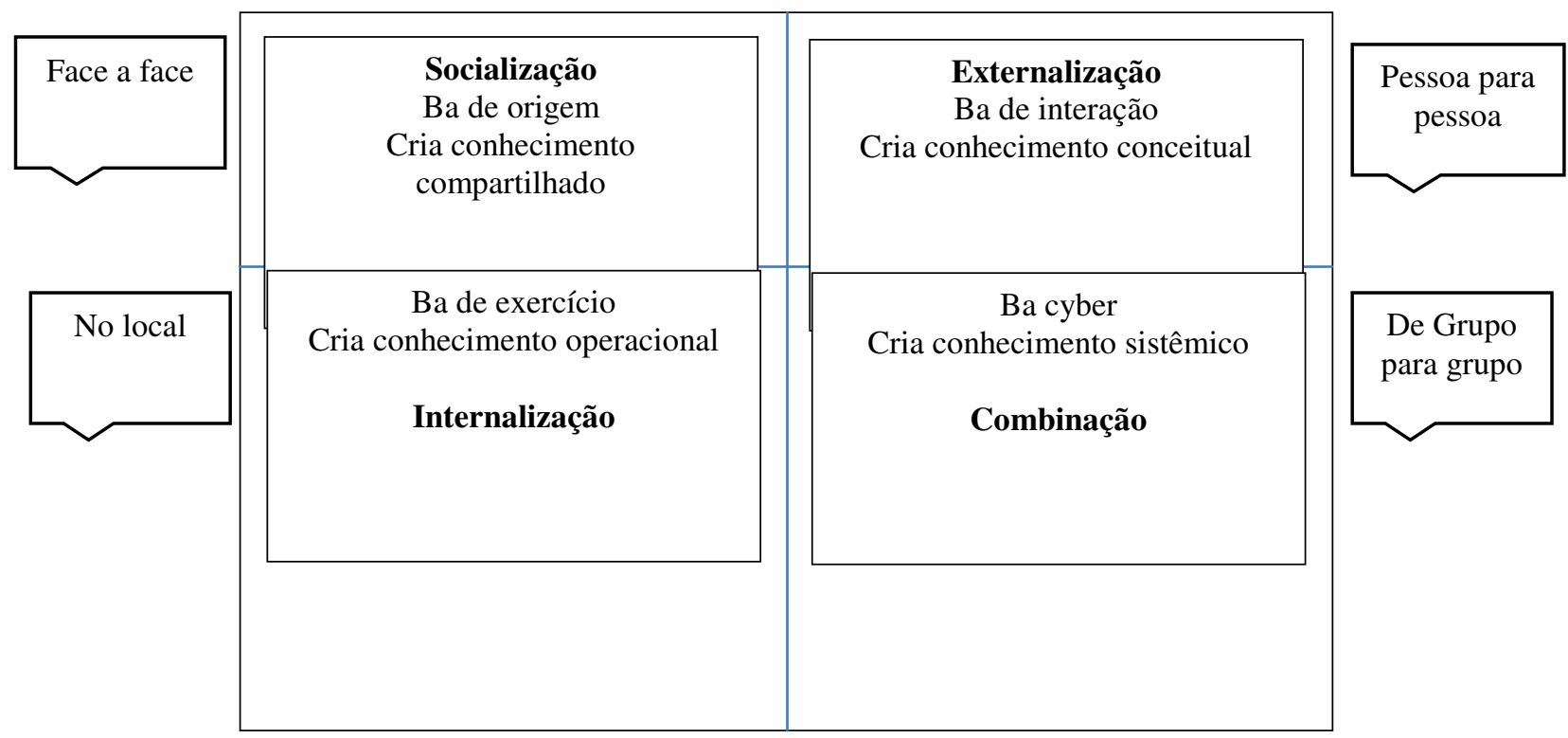

Fonte: NONAKA; KONNO (1998). 
Em primeiro lugar, os indivíduos retêm informações com base em suas próprias experiências e observações diretas. A segunda é a cultura organizacional, que é definida como uma forma concebida de perceber, pensar e sentir sobre os problemas que são transmitidos aos membros da organização (INAZAWA, 2009). O terceiro é a transformação, definida como a lógica que orienta a transformação de uma entrada em uma saída. O quarto é a estrutura, que diz respeito à forma como os indivíduos e grupos se relacionam internamente à empresa e em relação ao seu ambiente. A estrutura reflete e armazena informações sobre percepção do ambiente da organização. Por fim, a ecologia trata do local de trabalho, que tem um eficiente papel na retenção de informações sobre uma organização e seus membros.

Nesta pesquisa, estes dois modelos são utilizados para analisar o processo de retenção de conhecimento em uma operação de serviço. O modelo SECI de Nonaka e Takeuchi (1995) mostra o fluxo de conhecimento entre indivíduos, grupos e organização, e também identifica o estado de mudança de conhecimento (de tácito para explícito e de explícito para tácito).

O processo de retenção do conhecimento propõe um fluxo semelhante entre os indivíduos, grupos e organização, e também o processo de conversão entre o estado tácito e explícito. A partir da definição Walsh e Ungson (1991), o processo de retenção consiste em atividades de aquisição de conhecimento através do processo de socialização entre os indivíduos que compartilham o mesmo ambiente; armazenamento do conhecimento, apoiado pela externalização do conhecimento, no qual o conhecimento tácito do funcionário é articulado nos grupos e transformado em explícito, e ainda a combinação, por meio da qual o conhecimento explícito do grupo é sintetizado e aplicado; e, finalmente, a recuperação do conhecimento é sustentada pela internalização, na qual a memória organizacional é compartilhada e transformada novamente em conhecimento tácito entre os indivíduos. A partir do conhecimento tácito retido na memória organizacional e disponibilizado aos empregados, novos conhecimentos são criados.

\section{Método de pesquisa}

Determinadas dimensões culturais só devem ser estudadas a partir de uma abordagem qualitativa, ao analisar os aspectos sociais de uma rede de conhecimento (SCHEIN, 1999). As principais características dessa abordagem, que se encaixam nesta pesquisa, é o caráter interpretativo sobre a organização, a flexibilidade na pesquisa e a proximidade com o objeto de estudo (BRYMAN, 1989). 
Figura 3 - A estrutura da memória organizacional

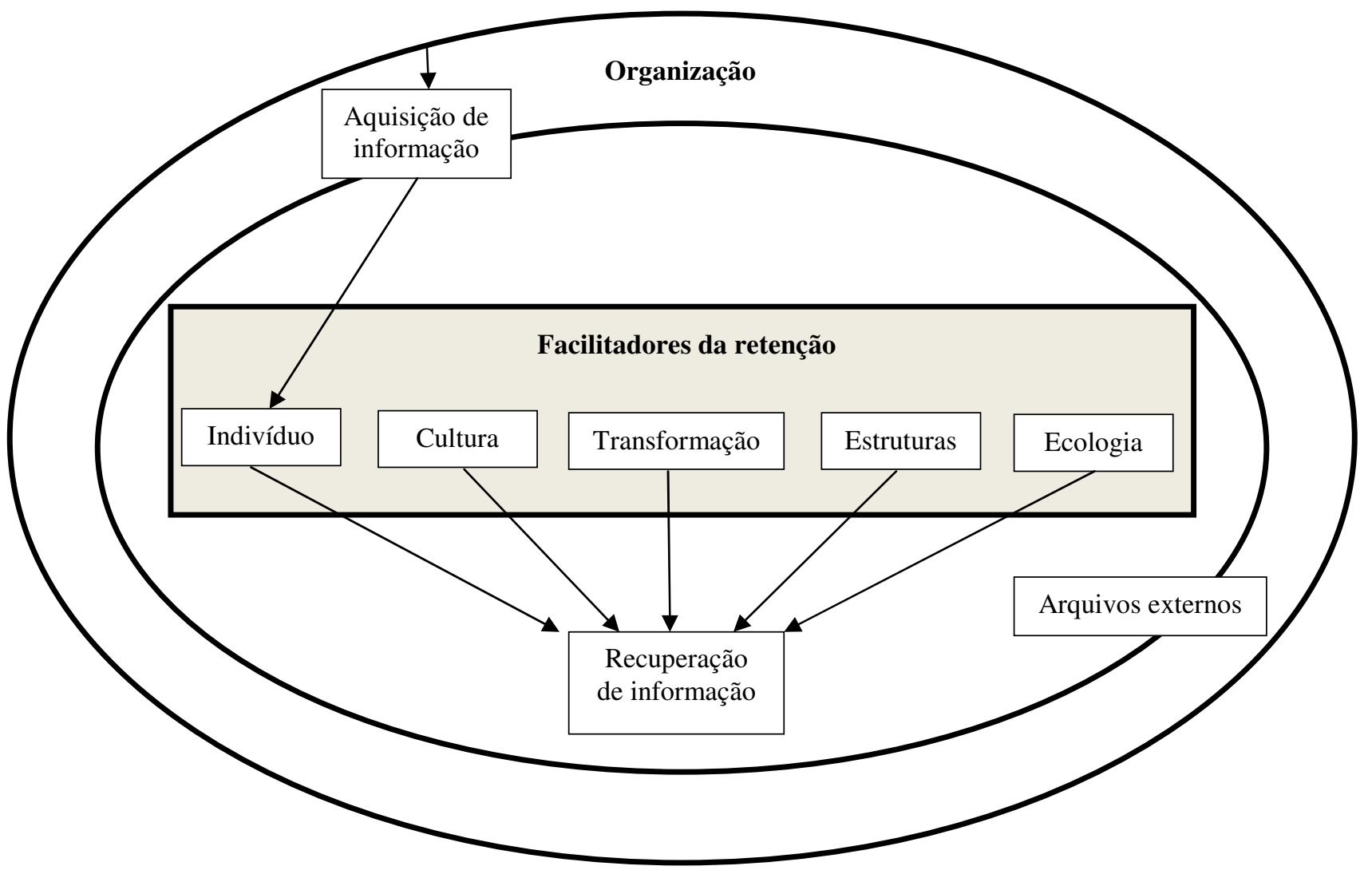

Fonte: WALSH; UNGSON (1991).

O método de estudo de caso foi considerado a estratégia de investigação mais adequada, pois, de acordo com Yin (2009) e Eisenhardt (1989), por meio dela, múltiplas dimensões de uma teoria pode ser estudado a fundo em um caso real. O procedimento de estudo de caso começa com o projeto de pesquisa e definição do objeto de estudo. A estratégia de estudo de caso adotado é o caso simples. Esta estratégia de investigação é aplicada quando se aplica uma exploração ampla e caracterização do objeto de estudo e quando suas características não são facilmente encontradas em outras amostras, e também na investigação de fenômenos contemporâneos dentro de um contexto real (YIN, 2009).

Yin (2009) define quatro questões a serem incluídas em um projeto de pesquisa de estudo caso:

1 Questão de pesquisa. Como um prestador de serviços gerencia o processo de retenção do conhecimento, a fim de minimizar a perda de conhecimentos adquiridos em diversos sites de prestação de serviço?

2 Proposição de pesquisa. A pesquisa propõe um modelo de gestão que permite a uma empresa prestadora de serviços industriais reter, e também recuperar os conhecimentos adquiridos em vários clientes. 
3 Unidades de análise. A unidade de análise da pesquisa é a organização. A organização selecionada é um prestador multinacional de serviços industriais, instalada no Brasil com matriz sueca. A seleção de uma única empresa é explicada pela busca de profundidade na coleta de dados e análise. Além disso, o tipo de organização pesquisada (prestador de serviços que opera em diferentes clientes industriais) não é facilmente acessado. O processo de retenção de conhecimento na empresa pesquisada envolve duas áreas: Sites Full service e Centro de Excelência. Os sites full service representam as unidades de prestação de serviços, a "linha de frente" da empresa, que apresentam alto contato com os clientes. O centro de excelência representa o núcleo da estrutura de gestão do conhecimento da empresa pesquisada. Nele, ocorre o processo de validação e armazenamento de conhecimento para posterior distribuição. As características da empresa são descritos em maiores detalhes na seção seguinte. As pessoas incluídas no estudo foram os gestores de três sites full service, que prestam serviços a empresas do segmento de bebidas, e também um gerente responsável pelo Centro de Excelência da organização estudada. As entrevistas foram realizadas durante o segundo semestre de 2013.

A lógica que liga os dados para a proposição e os critérios para a interpretação dos resultados. O questionário da pesquisa foi estruturado em torno de duas partes principais. A primeira trata das características essenciais da organização, tais como número de funcionários, quantidade de sites full service, os setores dos sites full service, número de centros de excelência, etc. A segunda parte, composta por dezoito questões, trata dos aspectos de retenção de conhecimento adotados pela organização. A fim de validar o questionário, ele foi submetido a um teste piloto realizado com dois pesquisadores da área de GC e aprendizagem organizacional e outros dois profissionais de organizações que adotam o processo de GC. O teste-piloto teve como objetivo verificar o nível de compreensão das questões pelos participantes do teste. Descobrimos que, das dezoito questões formuladas, duas tinham problemas de compreensão, e foram modificadas a fim de promover uma melhor compreensão. Os dados foram analisados em torno de três aspectos importantes da retenção do conhecimento (aquisição, armazenamento e recuperação). Além disso, o modelo SECI de Nonaka e Takeuchi e o modelo de memória organizacional de Walsh e Ungson (1991) são utilizados para a análise do processo de retenção do conhecimento da empresa prestadora de serviços. A análise desses dados nos permitiu estruturar a seção "Análise e Resultados".

\section{Evidências empíricas}

\subsection{Caracterização da empresa}

A empresa estudada é uma subsidiária brasileira de uma empresa multinacional europeia. O foco desta pesquisa é a área de serviços industriais, que representa um lucro líquido mundial de cerca de U\$ 100 
milhões / ano, empregando cerca de 60.000 funcionários, com a missão de garantir serviços de alto valor agregado e de trabalhar em parceria com os seus clientes. No Brasil, a unidade de serviços industriais representa um lucro líquido de cerca de U\$8 milhões dólares / ano e emprega cerca de 4.500 funcionários.

A empresa identifica o full service como o seu principal produto, que pode ser definido como um contrato de longo prazo, com foco na prestação de serviços nas instalações industriais de seus clientes, a fim de aumentar sua produtividade e performance, trabalhando com técnicas sofisticadas na área de engenharia industrial, processos, manutenção e automação.

Os contratos de full service são chamados de sites de prestação de serviços, que constituem a base da pirâmide hierárquica ao analisar o organograma da empresa pesquisada, sendo composto por grupos de funcionários de linha de frente, localizados dentro da área industrial dos diferentes clientes, apresentando uma relação bastante intensa com estes.

Atualmente, a empresa pesquisada tem, no Brasil, uma carteira de 57 contratos full service, dividido em seis segmentos (Tabela 2). Este agrupamento de clientes possibilita a formação de portfólios com uma estrutura organizacional por cliente.

Tabela 2 - Segmentos de atuação da empresa pesquisada

Fonte: Dados da pesquisa.

\begin{tabular}{cc}
\hline Segmento & Quantidade \\
\hline Eletrônico & 15 \\
Petroquímico & 13 \\
Alimentos & 13 \\
Papel e Celulose & 6 \\
Bebidas & 5 \\
Metalurgia & 5 \\
\hline
\end{tabular}

Esta divisão de clientes por segmentos de atuação é uma forma de agrupar os clientes com processos industriais similares, formando carteiras que compartilham recursos (trabalho, máquinas, equipamentos) e, em particular, o conhecimento.

\subsection{Estrutura para retenção do conhecimento}

A estrutura organizacional da empresa para a retenção do conhecimento é caracterizada por grupos de trabalho estabelecidos nos sites full service que interagem com o Centro de Excelência. Neste trabalho, a análise desta estrutura é realizada através do método de estudo de caso.

\subsubsection{Sites full service}

Os sites ful service são coordenados pelas administrações Full service do respectivo segmento, e dentro de cada site há um gestor de contrato, que coordena uma série de divisões funcionais: engenharia industrial, engenharia de manutenção, planejamento e controle de 
manutenção, oficinas e supervisores responsáveis pelo trabalho operacional.

Normalmente, os contratos de serviços full service são acordados em torno de dois valores. O primeiro corresponde a um valor fixo a ser pago pelo cliente, dependendo da extensão dos processos e serviços contratados. A segunda parte refere-se a um contrato de produtividade. Com isso, a empresa prestadora de serviço aumenta a sua receita mensal a partir da implementação de ações que resultem em melhoria de eficiência. A partir de um processo de medição de desempenho que indica o aumento da produtividade, o cliente paga ao prestador de serviço um percentual de ganhos financeiros. Assim, estabelece-se uma situação ganha-ganha, na qual ambas as partes são beneficiadas, melhorando o desempenho do processo.

Os funcionários de cada um dos setores dos sites full service são responsáveis por atividades de rotina, estabelecidas no âmbito do contrato, e por atividades de melhoria de produtividade. As atividades operacionais desenvolvidas nos três sites pesquisados são: elaboração e revisão dos processos de produção, desenvolvimento e revisão de planos de manutenção preventiva, cumprimento do plano de manutenção preventiva, ações corretivas, suporte operacional, aquisição de máquinas e ferramentas.

Há um incentivo e uma organização da empresa pesquisada para formalizar todos os processos e atividades técnicas realizadas dentro dos sites full service. Os processos são registrados em processos administrativos (PAs), que representam os padrões para as atividades que ocorrem nos contratos, como, por exemplo, sistemática para o desenvolvimento de planos de manutenção, para a solicitação de serviços de engenharia ou de consultoria, para a contratação de funcionários, para a compra de materiais, entre outros.

Atividades técnicas, por sua vez, são documentadas em instruções técnicas (ITs). Nestas, são relatadas todas as medidas necessárias para o desenvolvimento das atividades operacionais, seja para manutenção, operação, configuração de máquinas, entre outros, e também relacionadas com a melhoria de um processo.

Assim, pode-se afirmar que os PAs estão em um nível mais macro e ITs estão em um nível menor, uma vez que o próprio estabelecimento de uma IT é uma atividade padronizada, descrita em um AP.

As instruções técnicas desempenham um papel fundamental no processo de GC, pois estas explicitam todas as atividades realizadas em um site full service. As ITs são periodicamente auditadas pela equipe do Centro de Excelência em três aspectos:

1 As atividades estão sendo realizadas no âmbito de sua respectiva IT?

2 Os funcionários dos sites full service revisam as ITs quando promovem uma mudança nos procedimentos de execução? 
3 Os funcionários dos sites full service desenvolvem novas ITs quando novas atividades ou melhorias são realizadas?

Com relação às atividades de melhoria, as equipes dos contratos, sob a coordenação dos gestores operacionais, realizam as seguintes atividades:

1 Kaizen: reunião de funcionários de diferentes especialidades com a missão de resolver um problema específico, definido pelo gerente, que fornece os recursos necessários;

2 Projetos de engenharia: mudanças nos sistemas de produção, incluindo a reestruturação do processo de produção, automação, instalação de máquinas e manutenção de novos procedimentos, que envolve especificamente a equipe de engenharia dos sites full service;

3 Programas de melhoria autônoma: melhorias espontâneas propostas e/ou implementadas pelos funcionários dos sites, caracterizadas por pequenas iniciativas;

4 Iniciativas da rede full service: propostas de melhoria decorrente da base de dados intranet, na qual são alocadas as informações de todos os projetos de melhoria realizados nos sites full service com o apoio do Centro de Excelência para a sua implementação.

Além desses programas citados para promover a criação de novos conhecimentos através de trabalho em equipe multidisciplinar, os sites full service também recebem a assessoria técnica de consultores internos da empresa, em particular, nas atividades de melhoria que envolvem conhecimentos originados em outros sites. Assim, o consultor atua como um multiplicador do conhecimento tácito desenvolvido em determinado site.

Dependendo do escopo do contrato, a empresa pesquisada pode participar ou não dos resultados obtidos com as melhorias implementadas. Os três sites pesquisados recebem uma parte do lucro, após a conclusão de uma melhoria que trouxe resultados positivos significativos para 0 cliente, cujos valores percentuais não foram fornecidos pelos entrevistados. A partir desta participação, uma porcentagem é atribuída ao grupo de funcionários que trabalharam na melhoria a fim de estimular a busca constante por melhorias, e outra é direcionada ao resultado operacional do site.

\subsubsection{Centro de Excelência}

Também conhecida como a área de GC da empresa pesquisada, tem a mesma divisão por segmentos estabelecidos nos sites full service 
(Tabela 2), coordenado por supervisores responsáveis por funcionários que atuam no processo de GC. Cada Centro de Excelência tem um número de funcionários que varia com o tamanho da carteira de clientes e do tamanho dos contratos de full service. No caso do segmento de bebidas, o centro de excelência tem sete funcionários que trabalham nas seguintes atividades:

a. Identificação: o Centro de Excelência mantém comunicação constante com os funcionários que estão desenvolvendo melhorias nos sites full service, seja técnica (por exemplo, a automação de um dispositivo), ou metodológico (por exemplo, a reformulação de um procedimento para reduzir o tempo de setup de uma linha de produção). As equipes de engenharia dos sites full service têm a responsabilidade de acrescentar informações referentes a qualquer melhoria que esteja em curso na base de conhecimento que a empresa pesquisada disponibiliza aos funcionários e que é gerida pelo Centro de Excelência;

b. Avaliação: no momento em que uma melhoria é implementada, os funcionários do Centro de Excelência do segmento ao qual o site pertence são chamados para medir os resultados, avaliando se o método utilizado resultou em fracasso ou sucesso, a partir dos seguintes indicadores:

- Financeiro: usado quando a melhoria implementada impacta os custos do processo;

- Tempo de processamento: usado quando uma melhoria implementada impacta no tempo de execução de uma atividade de determinado processo;

- Qualidade: usado quando uma melhoria implementada impacta em algumas características do processo, tais como a qualidade do produto, ergonomia, segurança, entre outros, em uma escala de 1 (abaixo do resultado esperado) a 5 (resultado muito satisfatório);

c. Armazenamento: Depois de avaliar os resultados da melhora, o Centro de Excelência desenvolve um relatório, em conjunto com a equipe do site full service, contendo todos os passos de uma melhoria específica;

d. Distribuição: os relatórios de novos procedimentos e melhorias da fase anterior, bem como as suas avaliações, estão disponíveis para os funcionários de outros sites ou centros de excelência através de um banco de dados na intranet. Além de facilitar o acesso a esses documentos, os Centros de Excelência também fornecem uma equipe para auxiliar a implementação das melhorias.

\subsection{Rede de cooperação entre sites e centros de excelência}

Embora cada site possua uma equipe que à primeira vista atua de forma isolada, longe dos outros membros da organização pesquisada, na realidade, eles estão em constante contato com outros funcionários de outros sites, especialmente, aqueles que pertencentes ao mesmo segmento. 
Funcionários de cada site full service trabalham em atividades de melhoria, muitas vezes em parceria com os membros da empresa cliente, o que proporciona o desenvolvimento de novos conhecimentos em relação aos processos envolvidos.

A empresa pesquisada coloca os sites full service em uma estrutura em rede, facilitando a comunicação e interação entre as pessoas que trabalham nos clientes, especialmente aqueles do mesmo segmento. A comunicação e a integração dos funcionários de diferentes sites são asseguradas pelo centro de excelência. O centro de excelência de um segmento específico, como o segmento de bebidas do caso estudado nesta pesquisa, tem como objetivo identificar as melhores práticas desenvolvidas em cada site full service e disseminar o conhecimento entre os outros sites do mesmo segmento.

Fazendo uma analogia com a química, os sites full service representam átomos, a menor unidade de estrutura para a retenção de conhecimento, que estão alinhados em torno dos Centros de Excelência do respectivo segmento, representando moléculas. A estrutura, como um todo, constituída de vários sites, orientada pelos centros de excelência, representa uma célula, isto é, a empresa pesquisada.

Embora cada site possua um grupo de funcionários da empresa pesquisada que a primeira obra trabalha de forma isolada, longe da estrutura, na realidade, eles estão em constante contato com outras pessoas de outros sites, especialmente aqueles referentes ao mesmo portfólio e alinhados por objetivos organizacionais comuns.

Uma das principais funções do Centro de Excelência é proporcionar novos conhecimentos a todos os outros sites full service por meio de:

a) Banco de dados de conhecimento;

b) Documentos (procedimentos administrativos e instruções técnicas);

c) Deslocamento de funcionários entre os sites full service;

d) Disponibilização de consultores dos Centros para a Excelência e Engenharia para implementar uma melhoria, um novo procedimento ou treinamento;

e) Fóruns entre os funcionários que estejam atuando em projetos de melhoria em diferentes sites full service.

Além da identificação e armazenamento do conhecimento desenvolvido pelos sites full service, o Centro de Excelência tem equipe de consultores, formada por técnicos e engenheiros que dominam as competências relacionadas a um segmento específico. Por exemplo, o centro de excelência no segmento de bebidas, considerado neste estudo, tem um grupo técnico que detém conhecimentos e habilidades sobre 0 processo de envase de líquido. Assim, este grupo de funcionários, a partir da experiência vivida em cada site full service, possui domínio técnico sobre 0 processo de envase, as características das máquinas, 
procedimentos de trabalho, planos de manutenção preventiva e melhores práticas.

Os centros de excelência de diversos segmentos são coordenados por um Centro de Excelência Matriz, representado no centro da Figura 4, que coordena a ação de todos os centros de excelência dos diversos segmentos. Suas principais responsabilidades estão relacionadas com a gestão de todos os centros de excelência:

a) estabelecimento de metas para cada centro de excelência;

b)contratação e treinamento dos funcionários envolvidos com as atribuições dos centros de excelência;

c) disponibilidade de banco de dados para armazenamento de conhecimento;

d) a gestão financeira do setor.

Uma deficiência observada pelos gestores da empresa pesquisada encontra-se na interação entre os centros de excelência em diferentes segmentos e, consequentemente, na interação dos sites dos próprios de diferentes segmentos. Embora o banco de dados seja único, permitindo a consulta de todas as informações por qualquer site ou centro de excelência, a interação entre os funcionários de sites pertencentes a diferentes segmentos não ocorre com tanta frequência.

\subsection{Análise e resultados}

Inicialmente, ao analisar o processo de retenção de conhecimento da empresa pesquisada, é possível destacar que parte do conhecimento adquirido nos sites full service não pode ser efetivamente convertido em conhecimento explícito, sem perda de significado e contexto.

Também vale a pena ressaltar que o valor do conhecimento somente é preservado quando é mantido em um estado não convertido, ou seja, no estado tácito através de indivíduos e grupos dentro da organização. Assim, a principal contribuição que a análise do estudo de caso deste artigo propõe consiste em avaliar o processo de retenção do conhecimento, destacando os mecanismos utilizados por um prestador de serviço multi-site para a retenção não apenas do conhecimento explícito, mas também do conhecimento tácito.

A análise do processo de retenção de conhecimento identificado na empresa pesquisada é realizada a seguir e assume que a retenção do conhecimento envolve a aquisição, armazenamento e recuperação de conhecimento em um processo cíclico e contínuo, cujos limites entre os processos não são tão exatos (Walsh e Ungson, 1991). A análise é realizada a partir do modelo SECI de criação do conhecimento proposto Nonaka e Takeuchi (1995). 


\subsubsection{Aquisição de conhecimento: socialização (do indivíduo para o indivíduo)}

A primeira etapa envolve a socialização e compartilhamento de conhecimento no nível individual nos sites de prestação de serviços. Cada site estimula a criação de novos conhecimentos por meio de mecanismos de aprendizagem, em particular grupos de resolução do problema e de melhoria, que promovem a interação entre os indivíduos e a disseminação do conhecimento tácito. O gerente do site tem um papel fundamental nesta fase, pois ele é responsável em desenvolver um ambiente que promova a criação e integração entre os indivíduos. Dentro dos sites de prestação de serviços, os indivíduos constroem um senso de confiança e identidade que facilita o diálogo e interação interpessoal. Este tipo de relação entre as pessoas facilita o desenvolvimento de grupos de trabalho autogeridos nos sites. A sinergia criada dentro destes grupos promove o desenvolvimento de uma base de conhecimento primário que facilita o fluxo de conhecimento tácito entre os indivíduos.

É possível considerar que cada site full service atua como verdadeiros "laboratórios" para a empresa pesquisada. Nestes ambientes, a empresa pode promover um processo de experimentação, explotação e exploração de novos conhecimentos, a partir de uma base de conhecimento primário dominada pelos clientes. Os contratos firmados entre a empresa pesquisada e os clientes também promovem a busca por melhoria e inovação, gerando novos conhecimentos. Esses contratos são geralmente estabelecidos em torno de ganhos alcançados por meio do aumento da produtividade. Assim, quanto mais melhorias a empresa traz para o seu cliente, mais retorno financeiro ela alcança, bem como seus funcionários que ganham recompensas e prêmios a partir das melhorias implementadas.

\subsubsection{Armazenamento do conhecimento: externalização e combinação (do indivíduo para o grupo e do grupo para a organização)}

Esta etapa envolve a conversão do conhecimento tácito em conhecimento explícito. O maior desafio no contexto da prestação de serviço multi-site é reter o conhecimento tácito adquirido em diferentes contextos e subculturas de pequenos grupos que são mantidos nas empresas clientes. O conhecimento explícito criado deve ser uma reflexão das melhores práticas e lições aprendidas dentro dos clientes. O principal agente capaz de identificar as boas práticas e lições aprendidas, codificálas e torná-las disponíveis para toda a organização é o centro por excelência.

O centro de excelência também trabalha com os processos de retenção e distribuição de conhecimento tácito. Para atingir este objetivo, a empresa pesquisada tem funcionários chamados de "consultores do conhecimento" de processos específicos dos segmentos que a empresa 
atua. Esses consultores atuam na identificação e avaliação de conhecimento, seja explícito ou tácito. Uma vez que o conhecimento adquirido dentro de um site é avaliado positivamente, começa o processo de externalização (NONAKA; TAKEUCHI, 1995). Se o conhecimento é codificável, ele é armazenado no banco de dados do centro de excelência de seu segmento, que oferece esse conhecimento para todos os sites do segmento, e também para os centros de excelência dos outros segmentos. Esses consultores também atuam na retenção e disseminação do conhecimento tácito. Uma vez que nem todo o conhecimento é codificável, esses funcionários visitam outros sites a fim de disseminar e inserir uma determinada lição aprendida dentro de um novo contexto, ocorrendo, desta forma, o processo de combinação de conhecimento (NONAKA; TAKEUCHI, 1995). Além disso, os centros de excelência promovem a rotatividade de funcionários técnicos entre sites para disseminar as melhores práticas e, em especial, a fim de que o conhecimento tácito seja articulado entre os funcionários de outros sites em diferentes contextos. Assim, o centro de excelência age como a memória organizacional da empresa pesquisada.

\subsubsection{Recuperação do conhecimento: internalização (da organização para o indivíduo)}

O processo de recuperação do conhecimento consiste na pesquisa e decodificação do conhecimento. Pesquisa é o processo pelo qual a informação retida é selecionada como relevante para determinado problema. Decodificação é a reconstrução da informação selecionada a fim satisfazer os requisitos do usuário (RODRIGUES; BLATTMANN, 2014; GAMMELGAARD; RITTER, 2005; ARIF et al., 2009). Assim, o conhecimento armazenado nos centros de excelência, que consiste na memória organizacional a empresa pesquisada, é disponibilizado a todos os sites e outros centros de excelência para utilização em melhorias e resolução de problemas inerentes aos processos dos clientes. Neste contexto, há um fluxo de conhecimento a partir do centro de excelência (organização) para os indivíduos de diferentes sites que a empresa presta serviço. Por meio deste processo, novos conhecimentos são desenvolvidos nos sites de prestação de serviços e absorvido pela organização por meio dos centros de excelência, expandindo a memória organizacional. A Figura 4 ilustra o processo de retenção do conhecimento através dos estágios de aquisição de conhecimento, armazenamento e recuperação.

\section{Considerações finais: discussões, limitações e trabalhos futuros}

O objetivo desta pesquisa foi analisar o processo de retenção do conhecimento adquirido no contexto de prestação de serviços multi-site. Um dos principais problemas enfrentados pelas empresas de serviços que têm equipes de trabalho que atuam nas unidades de seus clientes de manufatura consiste na identificação e retenção do conhecimento 
desenvolvido a partir de melhorias e atividades de resolução de problemas.

Uma vez que o conhecimento é desenvolvido em diferentes locais distantes da sede da empresa prestadora de serviço, torna-se essencial incluir um departamento que assuma a responsabilidade pela identificação, conservação, distribuição e recuperação do conhecimento. Este departamento, chamado de centro de excelência, tem a missão de centralizar 0 processo de retenção do conhecimento. O centro de excelência identifica as melhores práticas nos vários sites de serviço, mantém disponível para os funcionários dos vários sites, e também auxilia no processo de recuperação de conhecimento, ou seja, na aplicação do conhecimento nas diferentes áreas de serviço que a empresa pesquisada atua. Assim, o centro de excelência pode ser considerado o departamento que centraliza memória organizacional da empresa pesquisada.

A criação de contextos organizacionais que estimulem a interação social entre os funcionários é um aspecto importante para a criação de ambientes de aprendizagem. Em especial, quando a geração de melhorias deve ser constante, como nos sites pesquisados neste estudo, este contexto que valoriza a aprendizagem organizacional torna-se mais relevante para o desenvolvimento de indivíduos especialistas, que compartilham uma linguagem comum sobre os processos e problemas organizacionais. Como nem todo conhecimento pode ser explicitado, ou seja, migrado do estado tácito para o estado explícito, o desenvolvimento de ambientes que permitam uma grande interação individual é essencial para o processo de socialização do conhecimento (NONAKA; TAKEUCHI, 1995).

Figura 4 - Processo de retenção do conhecimento

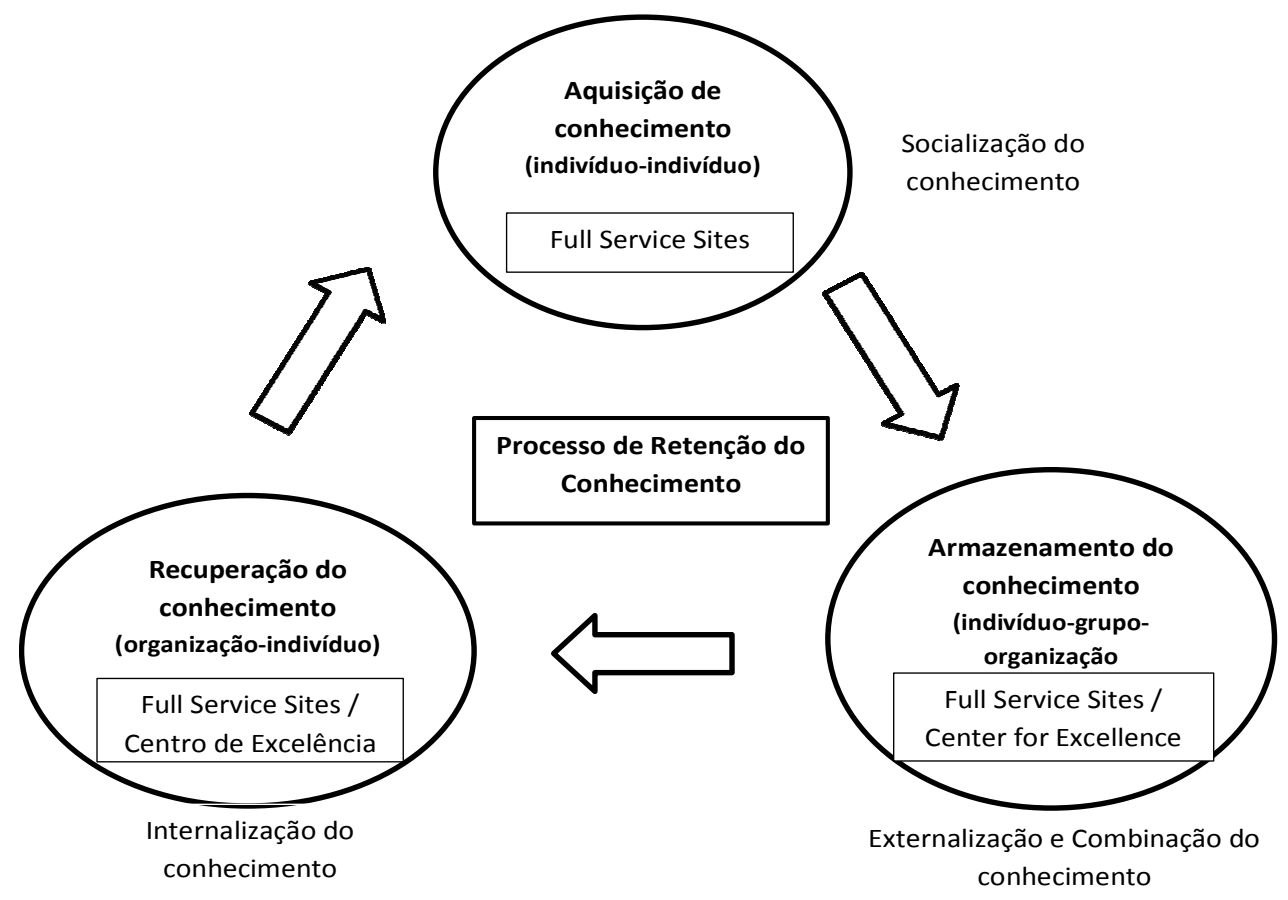

Fonte: Autoria própria 
Quanto ao processo que permite a retenção de conhecimento no contexto da prestação de serviço multi-site, as seguintes etapas foram identificadas a partir do estudo de caso: aquisição, armazenamento e recuperação do conhecimento.

A empresa pesquisada atua com dois agentes principais que sustentam o processo de retenção de conhecimento: sites full service e centros de excelência. Os sites full service são constituídos por equipes de funcionários que se dedicam inteiramente ao processo do cliente. Quanto ao processo de retenção do conhecimento, estas equipes trabalham na criação de conhecimento por meio de processo de resolução de problemas, melhoria incremental e inovações.

Os centros de excelência atuam como facilitadores do processo de retenção do conhecimento. Além de oferecer apoio técnico às equipes dos sites full service, os centros de excelência atuam no trabalho de mapeamento, avaliação, armazenamento e distribuição do conhecimento. Assim, a função principal dos centros de excelência é garantir que o conhecimento adquirido e transformado nos sites de prestação de serviço seja absorvido pela organização, ou seja, preservar o conhecimento organizacional da empresa prestadora de serviço.

Com relação às limitações desta pesquisa, o primeiro ponto a ser destacado é que a organização selecionada para o estudo é muito avançada em termos de gestão do conhecimento, produzindo resultados excessivamente positivos. Outro aspecto negativo está relacionado com a seleção metodológica. Embora o estudo de caso único possibilite uma ampla exploração das variáveis analisadas, este método não permite a extrapolação dos resultados para uma população maior.

Tendo em vista as limitações deste estudo, uma possível pesquisa futura seria a realização de mais análises aprofundadas de outras empresas que não conseguiram organizar um modelo adequado à retenção do conhecimento, investigando quais os aspectos mais críticos para estas empresas. Outra proposta consiste em uma análise comparativa das características estruturais que sustentam a retenção do conhecimento em diferentes setores, bem como organizações de diferentes nacionalidades. E, finalmente, a última proposta trata da análise das características estruturais das organizações através de uma abordagem metodológica quantitativa, baseada em técnicas de estatística multivariada.

\section{Referências}

ALAVI, M.; LEIDNER, D. E. Knowledge management and knowledge management systems: conceptual foundations and research issues. MIS Quarterly, v. 25, n. 1, p. 107-136, 2001.

ARGOTE, L.; MCEVILY, B.; REAGANS, R. Managing knowledge in organizations: An integrative framework and review of emerging themes. Management Science. V. 49, n. 4, p. 571-582, 2003. 
ARIF, M. Measuring knowledge retention: a case study of a construction consultancy in the UAE. Engineering, Construction and Architectural Management, v. 16, n. 1, p. 92-108, 2009.

BROWN, J. S.; DUGUID, P. Knowledge and organization: a social-practice perspective. Organization Science, v. 12, n. 2, p. 198-213, 2001.

BRYMAN, A. Research methods and organization studies. London: Unwin Hyman, 1989.

CLAVER, E., ZARAGOZA, P.; QUER, D. Practical experiences in knowledge management processes by multinational firms: a multiple case study'. International Journal of Knowledge Management Studies, v. 1, n. 3/4, p. 261-275, 2007.

DELONG, D. W.; DAVENPORT, T. Better practices for retaining organizational knowledge: lessons from the leading edge. Employment Relations Today, v. 30, n. 3, p. $51-63,2003$.

DU PLESSIS, F. New era organisations: from task-driven to customer-driven and world-class'. In: MOERDYK, A.; VAN AARDT, C. (Eds.). Organisational development: new methods and models for Southern Africa. South Africa: New Africa Books, p. 91-109, 2003

DURST, S.; EDVARDSSON, I. R. Knowledge management in SMEs: a literature review. Journal of Knowledge Management, v. 16, n. 6, p. 879903, 2012.

EDVARDSSON I. R.; OSKARSSON G. K. Knowledge management and value creation in service firms. Measuring Business Excellence, v. 15, n. 4, p. 8-15, 2011.

EISENHARDT, K. M. Building theories form case study research. Academy of Management Review, v. 14, n. 4, p. 532-550, 1989.

EVANSCHITZKY, H. et al. Knowledge management in knowledge-intensive service networks: a strategic management approach. Management Decisions, v. 45, n. 2, p. 255-283, 2007.

EVELETH, D. M. Knowledge management in changing environments: lessons from expert and non-expert decision-making literature. International Journal of Knowledge Management Studies, v. 4, n. 4, p. 375-388, 2011.

FILIPPINI, R. et al. The different modes for absorbing knowledge: an analytic lens on absorptive capacity from a process perspective. International Journal of Knowledge Management Studies, v. 5, n. 1/2, p. 45-65. 2012.

FREEZE, R. D.; KULKARNI, U. Knowledge management capability: defining knowledge assets. Journal of Knowledge Management, v. 11, n. 6, p. 94109, 2007.

GAMMELGAARD, J.; RITTER, T. The knowledge retrieval matrix: codification and personalization as separate strategies. Journal of Knowledge Management, v. 9, n. 4, p. 133- 43, 2005.

GAO, F.; LI, M.; CLARKE, S. Knowledge, management, and knowledge management in business operations. Journal of Knowledge Management, v. 12, n. 2 , p. $3-17,2008$. 
GARICANO, L.; WU, Y. Knowledge, communication, and organizational capabilities. Organization Science, v. 23, n. 5, p. 1382-1397, 2012.

GONZALEZ, R. V. D.; MARTINS, M. F. Mapping the organizational factors that support knowledge management in the Brazilian automotive industry. Journal of Knowledge Management, v. 18, n. 1, p. 152-176, 2014.

GRANT, R. M. Toward a knowledge-based theory of the firm. Strategic Management Journal, v. 17, n. 7, p. 109-122, 1996.

HUBER, G. Organizational learning the contributing processes and the literatures. Organizational Science, v. 2, n. 1, p. 88-115, 1991.

HUYSMAN, M.; WULF, V. IT to support knowledge sharing in communities, towards a social capital analysis. Journal of Information Technology, v. 21, n. 1, p. 40-51, 2006.

INAZAWA, F. K. O papel da cultura organizacional e da aprendizagem para - sucesso da gestão do conhecimento. Perspectivas em Ciência da Informação, v. 14, n. 3, p. 206-220, 2009.

LEE, C.; YANG, J. Knowledge value chain. The Journal of Management Development, v. 19, n. 9, p. 783-794, 2000.

LIN, H. F. A stage model of knowledge management: an empirical investigation of process and effectiveness. Journal of Information Science, v. 33, n. 6, p. 643-659, 2007.

MARTINS, E. C.; MEYER, H. W. J. Organizational and behavioral factors that influence knowledge retention. Journal of Knowledge Management, $\mathrm{v}$. 16, n. 1, p. 77-96, 2012.

McADAM, R.; MOFFETT, S.; PENG, J. Knowledge sharing in Chinese service organizations: a multi case cultural perspective. Journal of Knowledge Management, v. 16, n. 1, p. 129-147, 2012.

MOLINA, V. B.; AMADO, J. B.; AROSTEGUI, M. N. P. Managerial perceptions of the competitive environment and dynamic capabilities generation. Industrial Management \& Data Systems, v. 110, n. 9, p. 1355-1384, 2010.

NELSON, R.; WINTER, S. An evolutionary theory of economic change. Cambridge: Belknap Press/Harvard University Press, 1982.

NICHOLSON, N. Empower the next generation. Communication World, v. 25, n. 2, p. $14-18$, 2008. NICKERSON, J. A.; ZENGER, T. R. A knowledge-based theory of the firm: the problem-solving perspective. Organization Science, v. 15, n. 6, p. 617-632, 2004.

NONAKA, I.; KONNO, N. The concept of Ba': building a foundation for knowledge creation, California Management Review, v. 40, n. 3, p. 40-54, 1998.

NONAKA, I.; TAKEUCHI, H. The knowledge-creating company. New York: Oxford University Press, 1995..

POLANYI, M. The tacit dimension. London: Routledge \& Kegan Paul, 1967.

ROWLEY, J. Knowledge management in pursuit of learning. The learning with knowledge cycle. Journal of Information Science, v. 27, n. 4, p. 227$237,2001$. 
SILVA, A. B. O.; FERREIRA, M. A. T. Gestão do conhecimento e a teoria da firma. Perspectivas em Ciência da Informação, v. 14, n. 3, p. 116-139, 2009.

SMEDLUND, A. Social network structures for explicit, tacit and potential knowledge. International Journal of Knowledge Management, v. 5, n. 1, p. 78-87, 2009.

SPENDER, J. C. Making knowledge the basis of a dynamic theory of the firm. Strategic Management Journal, v. 17, p. 45-62, 1996.

RODRIGUES, C.; BLATTMANN, U. Gestão da informação e a importância do uso de fontes de informação para geração de conhecimento. Perspectivas em Ciência da Informação, v. 19, n. 3, p. 4-29, 2014.

TSOUKAS, $H$. The firm as a distributed knowledge system: a constructionist approach. Strategic Management Journal, v. 17, p. 11-25, 1996.

WALSH, P. J.; UNGSON, G. Organisational memory. The Academic of Management Review, v. 16, n. 1, p. 57 - 91, 1991.

YIN, R. K. Case study research: designs and methods. Los Angeles: Sage Publications, 2009. 